\title{
Deep sequencing exposes small RNA transcriptome differences between low- and high-temperature stress responses in Arabidopsis
}

\author{
Vesselin Baev, Ivan Milev, Mladen Naydenov, Tihomir Vachev, Elena Apostolova, Nikolay Mehterov, \\ Mariana Gozmanova, Ivan Minkov, Galina Yahubyan \\ University of Plovdiv, Plovdiv, Bulgaria
}

\section{Motivation and Objectives}

Plant small RNAs (sRNAs) include two major groups distinguished by their different modes of biogenesis - microRNAs (miRNAs) and small-interfering RNAs (siRNAs). Despite of numerous studies on the involvement of particular sRNA in plant stress response, there are only few reports on the genome-wide sRNA profiles generated under different stress treatments (Borsani et al., 2005; Yan et al., 2011; Katiyar-Agarwal et al., 2011). To understand the involvement of the various sRNA groups in plant response to different stress factors, we characterized sRNA datasets produced by highthroughput sequencing (HTS) from Arabidopsis plants suffering from low-temperature (LT) and high-temperature (HT) stress for 24 hour. Our study for the first time presents the genome-wide sRNA profiles of LT and HT treated plants and reveals that they greatly differed in the first 24 hours of stress onset.

\section{Methods}

Using Solexa technology for HTS that produces highly accurate and quantitative readouts of sRNAs, three sRNA libraries were generated and sequenced. The libraries derived from RNA extracted from leaf tissue of $A$. thaliana plants grown under normal temperature conditions $\left(21^{\circ} \mathrm{C}\right.$, NT library), and treated either with low temperature $\left(4^{\circ} \mathrm{C}\right.$, LT library) or high temperature $\left(36^{\circ} \mathrm{C}\right.$, HT library) for $24 \mathrm{~h}$. We obtained 21,364,883, $20,607,777$ and $22,542,401$ total reads for NT, LT and $H T$ library resp. After applying a quality-read filter and discarding low-quality reads, adaptor sequences were trimmed. Additionally, reads attributed to ligation contaminants or adaptor selfligation were also removed, that finally resulted in 19,881,084 (NT), 19,231,094 (LT) and 21,852,807 $(\mathrm{HT})$ clean reads. For the sRNA analysis, we further clustered all clean reads into unique sequences with associate copy numbers generating $2,134,578$ (NT), 1,534,898 (LT) and 3,308,837 (HT) unique tags for the three libraries.

\section{Results and Discussion}

Under the NT and LT conditions, the sRNA populations were dominated by 21-nt sRNAs. Another important finding is that there are many proteincoding genes that give rise to differentially expressed sRNAs following temperature shifts. The LT treatment caused increased production of sRNAs of sense polarity from numbers of cold-responsive genes such as COR15A, COR47, COR413, KIN2, KIN1. The HT treatment induced production of sRNAs of sense and antisense polarity from many genes encoding functionally diverse proteins. Amongst the temperature induced sRNAproducing loci, several can be selected whose sRNA profiles can be used to distinguish HTS Iibraries generated under low-temperature stress from those generated under high-temperature treatment.

\section{Acknowledgements}

This work was supported by FP7 BIOSUPPORT project.

\section{References}

Borsani O, Zhu J, Verslues PE, Sunkar R, Zhu JK (2005): Endogenous siRNAs derived from a pair of natural cis-antisense transcripts regulate salt tolerance in Arabidopsis. Cell. 123(7):1279-91.

Katiyar-Agarwal S, Morgan R, Dahlbeck D, Borsani O, Villegas A Jr, Zhu JK, Staskawicz BJ, Jin H. (2006): A pathogen-inducible endogenous siRNA in plant immunity., Proc Natl Acad Sci U S A. 103(47):18002-7.

Yan Y, Zhang Y, Yang K, Sun Z, Fu Y, Chen X, Fang (2011): Small RNAs from MITE-derived stem-loop precursors regulate abscisic acid signaling and abiotic stress responses in rice. R. Plant J. 65(5):820-8. doi:10.1111/j.1365-313X.2010.04467.x 